\title{
Characterization of Fasiglifam-Related Liver Toxicity in Dogs ${ }^{\llbracket}$
}

\author{
Akifumi Kogame, ${ }^{1}$ Yuu Moriya, Ikuo Mori, Liping Pan, Akio Morohashi, ${ }^{2}$ Takuya Ebihara, ${ }^{1}$ \\ Hideo Fukui, ${ }^{1}$ Yoshihiko Tagawa, ${ }^{1}$ and Leslie Z. Benet
}

Drug Metabolism and Pharmacokinetics Research Laboratories (A.K., Y.M., A.M., T.E., Y.T.) and Drug Safety Research Laboratories (I.M., H.F.), Pharmaceutical Research Division, Takeda Pharmaceutical Company Limited, Fujisawa, Kanagawa, Japan; Takeda

Development Center Americas, Inc., Deerfield, Illinois (L.P.); and Department of Bioengineering and Therapeutic Sciences, Schools of Pharmacy and Medicine, University of California San Francisco, San Francisco, California (L.Z.B.)

Received November 2, 2018; accepted February 7, 2019

\section{ABSTRACT}

Fasiglifam, a potent and highly selective agonist of $\mathrm{G}$ protein-coupled receptor 40, was developed for the treatment of type 2 diabetes mellitus. However, phase III clinical programs were terminated owing to liver safety concerns. Fasiglifam-related liver toxicity was also observed in repeat-dose dog toxicology studies, characterized by granulomatous inflammation with crystal formation in the liver and/or bile ducts. These histopathological changes were not observed in rat toxicology studies. Matrix-assisted laser desorption/ionization time-of-flight mass spectrometry analysis of dog liver sections obtained from a repeat-dose toxicology study indicated that the crystalline material in the affected dog liver contained fasiglifam and fasiglifam glucuronide (fasiglifam-G).
Nonclinical mechanistic studies indicated that after 14 days of repeated oral dosing with $\left[{ }^{14} \mathrm{C}\right]$ fasiglifam at $200 \mathrm{mg} / \mathrm{kg}$ per day to dogs, the concentrations of fasiglifam and fasiglifam- $G$ in the bile exceeded the solubility limit of these compounds in the bile (approximately $3000 \mu \mathrm{g} / \mathrm{ml}$ ). After single oral 2- and 200-mg/kg doses administered to rats and dogs, fasiglifam and fasiglifam-G concentrations in dog bile were 5- to 10-fold higher than those in rat bile for the same dose of fasiglifam, while the bile flow rate adjusted by body weight was 4- to 8-fold lower in dogs than in rats. High fasiglifam and fasiglifam-G concentrations in dog bile together with lower bile flow rate could cause crystal formation in dog bile, resulting in secondary granulomatous inflammation in the dog liver.

\section{Introduction}

Fasiglifam (TAK-875), a potent and highly selective agonist of $G$ protein-coupled receptor 40, was developed for the treatment of type 2 diabetes mellitus (T2DM). G protein-coupled receptor 40 is highly expressed in pancreatic beta cells, and its activation induces insulin secretion. The insulinotropic effect of fasiglifam is strictly glucose dependent (Tsujihata et al., 2011). Fasiglifam only enhanced insulin secretion in islet cells in the presence of high ambient glucose, which is essential for a desired safety profile for the treatment of T2DM in terms of hypoglycemia risk. In clinical studies, fasiglifam reduced blood glucose and glycated hemoglobin levels with a low risk of hypoglycemia in T2DM patients (Kaku, 2013). However, the clinical development of fasiglifam was terminated in phase III clinical trials because of liver safety concerns (Kaku et al., 2015, 2016). From the fasiglifam clinical data focusing on liver safety findings, one definite Hy's law case [alanine aminotransferase (ALT) or aspartate aminotransferase $>3 \times$ upper limit of normal) and total bilirubin $>2 \times$ upper limit of normal] and two near Hy's law cases were identified in fasiglifam-treated patients

\footnotetext{
${ }^{1}$ Current affiliation: Axcelead Drug Discovery Partners, Inc., Fujisawa, Kanagawa, Japan

${ }^{2}$ Current affiliation: Nemoto Science. Co., Ltd., Joso, Ibaraki, Japan

https://doi.org/10.1124/dmd.118.084889.

S This article has supplemental material available at dmd.aspetjournals.org.
}

(Marcinak et al., 2018). Fasiglifam-related liver toxicity was also observed in repeat-dose dog toxicity studies. Fasiglifam treatment in these studies resulted in liver toxicity characterized by elevation of plasma aspartate aminotransferase, ALT, alkaline phosphatase, and/or bilirubin. Associated histopathological changes were characterized as portal/periportal granulomatous inflammation with crystal formation (Supplemental Figs. 1 and 2). This toxicity was observed in a 4-week study at a dose of $1000 \mathrm{mg} / \mathrm{kg}$ per day. The toxicity measures were greater than those observed for a dose of $150 \mathrm{mg} / \mathrm{kg}$ per day in a 13 -week study and for a dose of $80 \mathrm{mg} / \mathrm{kg}$ per day in a 39-week study, indicating that the toxicity was both dose and duration dependent (Supplemental Table 1). Although elevations of serum bilirubin and ALT were observed in repeat-dose rat studies (Wolenski et al., 2017), no histopathological changes indicative of liver injury were observed in any rat studies even at the lethal dose of $2000 \mathrm{mg} / \mathrm{kg}$ per day.

Our previous study indicated that disposition and metabolism of fasiglifam were similar between human and animals (Kogame et al., 2018). Fecal excretion was the primary elimination route for unchanged fasiglifam in all species. In addition, no human-specific metabolites were noted, and all metabolites found in human were also observed in the nonclinical animal toxicology studies, suggesting that the fasiglifamrelated liver toxicity observed in humans and dogs might not have been caused by the production of specific metabolites. Moreover, since glucuronidation is the major fasiglifam metabolic elimination pathway, the glucuronide of fasiglifam [fasiglifam glucuronide (fasiglifam-G)]

ABBREVIATIONS: ALT, alanine aminotransferase; BDC, bile duct-cannulated; Bsep, bile salt export pump; $\mathrm{CL}$, clearance; $\mathrm{E}_{2} 17 \beta \mathrm{G}$, estradiol $17 \beta$ D-glucuronide; fasiglifam-G, fasiglifam glucuronide; HPLC, high-performance liquid chromatography; LSC, liquid scintillation counter; MALDI TOF MS, matrix-assisted laser desorption/ionization time-of-flight mass spectrometry; Mrp2, multidrug resistance-associated protein 2; MS/MS, tandem mass spectrometry; NOAEL, no observable adverse effect level; Oatp, organic anion transporting polypeptide; TCA, taurocholic acid; T2DM, type 2 diabetes mellitus. 
could be considered as a liver toxicity candidate metabolite in addition to unchanged fasiglifam.

Dog liver toxicity was observed before the phase III study. Therefore, we conducted the present study to support the clinical development of fasiglifam. We identified the composition of foreign body materials observed in dog liver obtained from the 39-week repeat-dose studies by using matrix-assisted laser desorption/ionization time-of-flight mass spectrometry (MALDI TOF MS) analysis. Furthermore, we used several in vitro and in vivo approaches to characterize the mechanism of fasiglifam-related dog liver toxicity. Nonclinical mechanistic studies included the following: 1) metabolic profiles of $\left[{ }^{14} \mathrm{C}\right]$ fasiglifam in the plasma and liver samples; 2) potential covalent binding of fasiglifam-G to plasma and liver proteins; 3) $\left[{ }^{14} \mathrm{C}\right]$ fasiglifam accumulation in the plasma and liver of dogs; 4) disposition and metabolism of $\left[{ }^{14} \mathrm{C}\right]$ fasiglifam in rats and dogs; 5) hepatic transporter studies of fasiglifam and fasiglifam-G; 6) concentrations of total radioactivity, fasiglifam, and fasiglifam-G in bile after single or repeated dosing of $\left[{ }^{14} \mathrm{C}\right]$ fasiglifam; 7) bile flow rate and bile $\mathrm{pH}$ in rats and dogs in the presence of $\left[{ }^{14} \mathrm{C}\right]$ fasiglifam; and 8) in vitro solubility study of fasiglifam and fasiglifam-G. Based on these studies, we predicted fasiglifam and fasiglifam- $G$ concentrations in human bile of T2DM patients receiving $50 \mathrm{mg}$ dose to justify the phase III studies. The potential risk of hepatotoxicity in humans was assessed as discussed herein.

\section{Materials and Methods}

\section{Chemicals and Biologicals}

Fasiglifam ([(3S)-6-(\{2',6'-dimethyl-4'-[3-(methylsulfonyl)propoxy]biphenyl3-yl\}methoxy)-2,3-dihydro-1-benzofuran-3-yl]acetic acid hemihydrate) and 2',6'-dimethyl-4'-[3-(methylsulfonyl)propoxy]biphenyl-3-carboxylic acid were synthesized by Takeda Pharmaceutical Company Limited (Kanagawa, Japan). Furthermore, [(3S)-6-(\{2',6'-dimethyl-4'-[3-(methylsulfonyl)propoxy]biphenyl3 -yl $\}\left[{ }^{14} \mathrm{C}\right]$ methoxy)-2,3-dihydro-1-benzofuran-3-yl]acetic acid hemihydrate $\left(\left[{ }^{14} \mathrm{C}\right]\right.$ fasiglifam $)$ and glucuronic acid conjugate of $\left[{ }^{14} \mathrm{C}\right]$ fasiglifam $\left(\left[{ }^{14} \mathrm{C}\right]\right.$ fasiglifam-G) were synthesized by Daiichi Pure Chemicals Co., Ltd. (Tokyo, Japan) and Quotient Bioresearch (Pharmaron UK Limited, Cardiff, UK), respectively. Dog bile was purchased from Kitayama Labes Co, Ltd. (Yamaguchi, Japan). Human bile was purchased from KAC Co., Ltd. (Kyoto, Japan) and Wako Pure Chemical Industries, Ltd. (Osaka, Japan). $\left[{ }^{3} \mathrm{H}\right]$ Estradiol $17 \beta$-D-glucuronide $\left(\mathrm{E}_{2} 17 \beta \mathrm{G}\right)$ and $\left[{ }^{3} \mathrm{H}\right]$ taurocholic acid $(\mathrm{TCA})$ were purchased from PerkinElmer, Inc. (Waltham, MA). $\left[{ }^{3} \mathrm{H}\right]$ Pravastatin sodium salt was obtained from American Radiolabeled Chemicals (St. Louis, MO). Unlabeled pravastatin sodium salt and cyclosporin A were purchased from Wako Pure Chemical Industries, Ltd. Membrane vesicles were purchased from GenoMembrane Co., Ltd. (Kanagawa, Japan). The products were prepared from purified plasma membrane isolated from $\mathrm{Sf} 9$ cells infected with baculovirus-expressing rat and dog multidrug resistance-associated protein 2 (Mrp2) or bile salt export pump (Bsep). Rat and dog cryopreserved hepatocytes were purchased from Life Technologies Corporation (Carlsbad, CA).

\section{Animals}

Eight-week-old male Sprague-Dawley rats weighing 251-282 g were purchased from Charles River Laboratories Japan, Inc., (Ibaraki, Japan). Male beagle dogs (7-11 months old, 8.41-11.2 kg) were purchased from Kitayama Labes Co., Ltd. They were fed laboratory chow [for rats: CR-LPF (Oriental Yeast Co., Ltd., Tokyo, Japan); for dogs: CD-5M (CLEA Japan, Inc., Tokyo, Japan) or Labo D Stock (Nosan Corporation, Yokohama, Japan)], had free access to water, and were housed in temperature- and humidity-controlled rooms $\left(18-28^{\circ} \mathrm{C}\right.$; $38 \%-82 \%$ ), with 12-hour light/dark cycles, for more than 1 week before use. All experiments involving animals were reviewed and approved by the Animal Care and Use Committee of Takeda Pharmaceutical Company Limited.

\section{MALDI TOF MS}

The composition of the foreign body material in the liver in the dog toxicity studies was determined by conducting direct mass spectrometric measurement of fresh-frozen sections of liver tissue containing the foreign body materials obtained from a dog showing hepatotoxicity in the 39-week oral toxicity study of fasiglifam at $150 \mathrm{mg} / \mathrm{kg}$ per day with a 13-week recovery period. Sections were cut at a thickness of $10 \mu \mathrm{m}$ with a cryostat microtome (CM1950; Leica Microsystems, Germany). The sections were placed onto conductive slide glasses (indium tin oxide-coated slide glass) and stored at $-80^{\circ} \mathrm{C}$ until MALDI TOF MS analysis. The tissue slices were equilibrated to room temperature for at least 20 minutes before analysis. The glass slides were placed onto a special AXIMA MS plate (Shimadzu Corporation, Kyoto, Japan) for tissue analysis and then immobilized using double-sided conductive tapes. Furthermore, $200 \mathrm{nl}$ of peptide solution $(10 \mathrm{nmol} / \mathrm{ml}$ angiotensin II in $0.1 \%$ trifluoroacetic acid and $10 \mathrm{nmol} / \mathrm{ml}$ adrenocorticotropic hormone $18-39$ in $0.1 \%$ trifluoroacetic acid) was spotted for external calibration by manually pipetting near the tissue slices, and then $200 \mathrm{nl}$ of matrix solution $(20 \mathrm{mg} / \mathrm{ml} 2,5$-dihydroxybenzoic acid in $50 \%$ acetonitrile $/ 0.1 \%$ trifluoroacetic acid for positive mode or $4 \mathrm{mg} / \mathrm{ml}$ 9 -aminoacridine in $70 \%$ methanol for negative mode) was manually spotted at the same position.

The tiny foreign body materials in the liver tissue slices were directly measured by microdispensing a trace amount of the matrix solutions onto the lesion sites containing the foreign body materials in the tissue slices. First, the location of the foreign body materials was observed by microscopy, and then a landmark was prepared in the proximity of that location on the tissue slices by using a needle or pen. The sample plates, on which glass slides were immobilized with doublesided conductive tape, were set on the stage part of a chemical printer, and then an image of the sample plate was acquired using a scanner attached to a chemical printer instrument. The print positions to microdispense the matrix solution onto tissue sections were determined based on the landmark on the obtained image. Three hundred picoliters of $20 \mathrm{mg} / \mathrm{ml}$ 2,5-dihydroxybenzoic acid in 50\% acetonitrile $/ 0.1 \%$ trifluoroacetic acid or $4 \mathrm{mg} / \mathrm{ml}$ 9-aminoacridine solution in $70 \%$ methanol were microdispensed onto the tissue sections at a total volume of $18 \mathrm{nl}$ on a single spot.

Matrix solution was microdispensed onto spots on each tissue slice (marked as s1, s2, and s4 in Fig. 1) as the matrix toward the foreign body materials. After matrix deposition, each matrix deposit on the tissue slices was observed by microscopy. The matrix deposit, including foreign body materials, was measured by analyzing the foreign bodies (these spots are indicated by the red letters with numbers in Fig. 1). The printed positions corresponding to the normal region are shown by black letters with numbers in Fig. 1.

Direct measurement was performed on the tissue slices by using MALDI-TOF MS. The text file of the print positional information was exported from the chemical printer, and then direct analysis of the printed positions was performed based on the $X$ and $Y$ coordinates from the text file. The peptide solution, which was manually pipetted onto the glass slides, was used as an external calibration. Furthermore, tandem mass spectrometry (MS/MS) experiments were performed for the significant ions observed in the foreign body materials. The parameters for collision-induced dissociation were optimized to effectively produce fragment ions.

\section{Rat Studies}

The metabolite profiling studies of fasiglifam were investigated in intact and bile duct-cannulated (BDC) male rats. After a single oral administration of $\left[{ }^{14} \mathrm{C}\right]$ fasiglifam (final specific activity: $4.19 \mathrm{MBq} / \mathrm{mg}$ ) at 2 and $200 \mathrm{mg} / \mathrm{kg}$ to the intact rats, blood and liver were collected at 1 hour (time to maximum concentration of fasiglifam in rats) (Negoro et al., 2010) and 8 hours after administration. The blood was collected from the abdominal aorta under anesthesia by using diethyl ether. The blood was immediately cooled under ice-chilled conditions, and the plasma was obtained by centrifugation at $8000 \mathrm{~g}$ at $4^{\circ} \mathrm{C}$. Subsequently, the total radioactivity in the plasma was measured. The residual plasma samples were used for the metabolite profiling study.

For the BDC rat study, the rats were cannulated under anesthesia with isoflurane. After the efflux of bile was confirmed, the $\left[{ }^{14} \mathrm{C}\right]$ fasiglifam formulation was administered into the duodenum of the rats at 2 and $200 \mathrm{mg} / \mathrm{kg}$ (final specific activity: $0.763 \mathrm{MBq} / \mathrm{mg}$ for $2 \mathrm{mg} / \mathrm{kg}$ and $0.00741 \mathrm{MBq} / \mathrm{mg}$ for $200 \mathrm{mg} / \mathrm{kg}$ ). After administration, the rats were housed in Bollman cages. Bile was collected at 1-hour intervals for 8 hours, and then $8-24$ hours under dry ice-chilled conditions. The total radioactivity in the bile was measured. The residual 


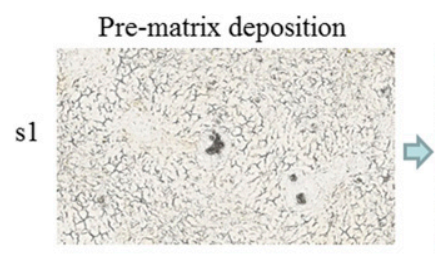

Post-matrix deposition
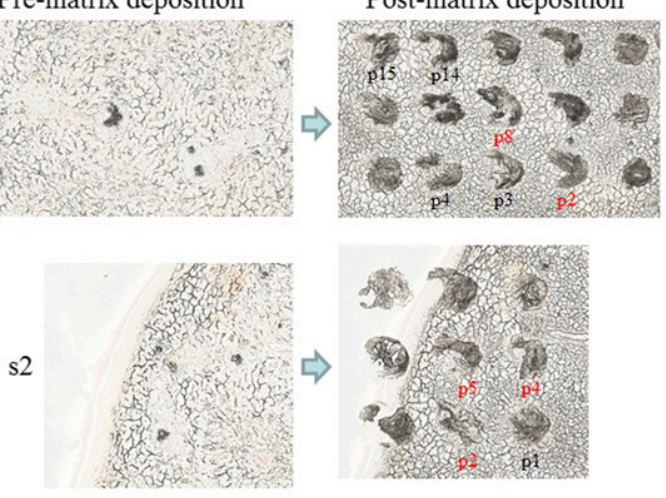
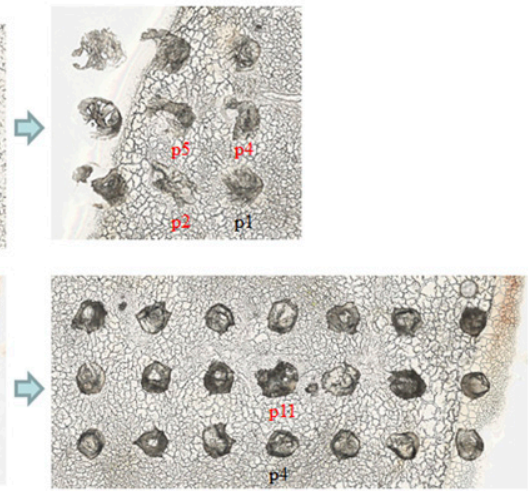

Fig. 1. Optical images of the matrix-deposited region on freshfrozen liver tissues. Letters in black: spots on the normal region; letters in red: spots on the foreign body material.

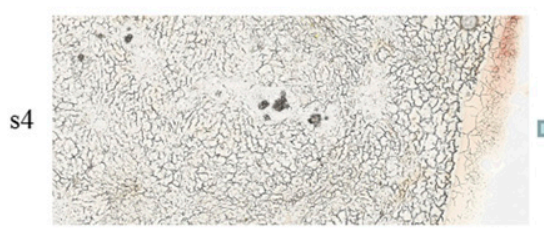

bile samples in each period were mixed together with the obtained weight ratio and used for the metabolite profiling study. The $\mathrm{pH}$ of the bile was measured using $\mathrm{pH}$ test paper (MACHEREY-NAGEL; Düren, Germany).

\section{Dog Studies}

The blood, liver, and bile samples were collected at 2 hours (time to maximum concentration of fasiglifam in dogs) (Negoro et al., 2010) and 8 hours after single oral doses of 2 and $200 \mathrm{mg} / \mathrm{kg}$ (final specific activity: $4.01 \mathrm{MBq} / \mathrm{mg}$ for $2 \mathrm{mg} / \mathrm{kg}$ and $4.19 \mathrm{MBq} / \mathrm{mg}$ for $200 \mathrm{mg} / \mathrm{kg}$ ) or 14-day repeated oral doses (final specific activity: $0.215 \mathrm{kBq} / \mathrm{mg}$ ) of $\left[{ }^{14} \mathrm{C}\right]$ fasiglifam at $200 \mathrm{mg} / \mathrm{kg}$ to intact dogs. The dogs were anesthetized with thiopental or sodium pentobarbital at an intravenous dose of $25 \mathrm{mg} / \mathrm{kg}$ for the collection of the liver and bile. During the repeated dosing study, the blood was collected at 5, 15, and 30 minutes and 1, 2, 4, 8, and 24 hours after the first, seventh, 13 th, and 14th administrations. The blood was collected from the cephalic vein. Blood samples were immediately cooled under ice-chilled conditions, and plasma was obtained by centrifugation at approximately $10,000 \mathrm{~g}$ at $4{ }^{\circ} \mathrm{C}$. Subsequently, total radioactivity in the plasma was measured. The gallbladder and liver were excised and immediately stored under ice-chilled conditions. Bile was collected almost entirely from the gallbladder by using syringes. The residual plasma, liver, and bile samples at 2 and 8 hours in the single dose study and after the 14th daily dose were used for metabolite profiling. The residual plasma samples in the repeated-dose study were used for the determination of fasiglifam concentration by using high-performance liquid chromatography (HPLC).

The time course of radioactivity in the bile after single oral administration of $\left[{ }^{14} \mathrm{C}\right]$ fasiglifam at doses of 2 and $200 \mathrm{mg} / \mathrm{kg}$ (final specific activity: $0.747-0.772 \mathrm{MBq} / \mathrm{mg}$ for $2 \mathrm{mg} / \mathrm{kg}$ and $0.00741 \mathrm{MBq} / \mathrm{mg}$ for $200 \mathrm{mg} / \mathrm{kg}$ ) was investigated in BDC dogs. The dogs were sedated with xylazine at a subcutaneous dose of about $3 \mathrm{mg} / \mathrm{kg}$, and anesthesia was maintained with isoflurane. Just before the operation, the dogs were given cefazolin as an intravenous dose of about $25 \mathrm{mg} / \mathrm{kg}$ and meloxicam at a subcutaneous dose of about $0.2 \mathrm{mg} / \mathrm{kg}$. During the operation, dextrose/acetate Ringer's solution was intravenously infused via the cephalic vein at a rate of about $100 \mathrm{ml} / \mathrm{h}$ per body. A cannula was inserted into the bile duct toward the liver, and another cannula was inserted into the bile duct toward the duodenum. The ends of the cannulas were interconnected on the dorsal region with a joint tube until dosing. After stable bile flow into the cannula was confirmed, $\left[{ }^{14} \mathrm{C}\right]$ fasiglifam was orally administered. Bile was collected at 1-hour intervals for 8 hours, and then at $8-24$ hours under dry ice-chilled conditions. The total radioactivity in the bile was measured. The residual bile samples in each period were mixed together with the obtained weight ratio and used for the metabolite profiling study. The $\mathrm{pH}$ of the bile was measured using a $\mathrm{pH}$ test paper (MACHEREY-NAGEL).

\section{Metabolite Profiling Studies}

The metabolite profiles in the plasma, bile, and liver obtained from single administration studies were elucidated as reported previously by using an online radioisotope detection method (Kogame et al., 2018). In brief, HPLC analyses were performed on a Shimadzu Prominence or LC-VP HPLC system (Shimadzu Corporation). The separation was performed on a reversed-phase HPLC column (Inertsil ODS-3; $4.6 \mathrm{~mm}$ i.d. $\times 250 \mathrm{~mm}, 5 \mu \mathrm{m}$; GL Sciences Inc., Tokyo, Japan) at a flow rate of $1 \mathrm{ml} / \mathrm{min}$. The column temperature was maintained at $40^{\circ} \mathrm{C}$. The HPLC eluate of the animal samples was mixed with Ultima-Flo AP (PerkinElmer, Inc.) at 1:3, and the radiolabeled components were quantified after background subtraction. The mobile phases were $0.01 \mathrm{~mol} / \mathrm{l}$ ammonium formate/acetonitrile/formic acid (900:100:1, v/v/v; solvent A) and $0.01 \mathrm{~mol} / \mathrm{l}$ ammonium formate/acetonitrile/formic acid (100:900:1, v/v/v; solvent B). The samples were eluted using the following gradients: $40 \%-50 \% \mathrm{~B}$, 0-45 minutes; $50 \%-100 \%$ B, 45-45.1 minutes; $100 \%$ B, $45.1-50$ minutes; $100 \%-40 \%$ B, 50-50.1 minutes; and $40 \%$ B, 50.1-60 minutes.

The metabolite profiles in the liver obtained from repeat dosing were elucidated using the offline HPLC method employing an ultra-low-background liquid scintillation counter (LSC) (Onishi et al., 2013). Fractions were collected every 1 minute, and the radioactivity was measured using an ultra-low-background LSC (1220 Quantulus; PerkinElmer, Inc.). The radioactivity in each elute was detected as described in Measurement of Radioactivity.

\section{Hepatobiliary Transporter Studies}

Uptake of $\left[{ }^{14} \mathbf{C}\right]$ Fasiglifam into Hepatocytes. Hepatic uptake of $\left[{ }^{14} \mathrm{C}\right]$ fasiglifam was investigated using cryopreserved hepatocytes from rat and dog (two donors for each species). Hepatocytes preserved in the gas phase of liquid nitrogen were thawed at $37^{\circ} \mathrm{C}$, and then suspended in recovery medium (High Viability CryoHepatocyte Recovery Medium; BD Biosciences, Franklin Lakes, NJ). The suspensions were mixed gently three times and centrifuged at approximately $100 \mathrm{~g}$ for 10 minutes at $23^{\circ} \mathrm{C}$. The supernatants were removed, and the residual hepatocytes were suspended in the plating medium (CryoHepatocyte Plating Medium; BD Biosciences). The cells were resuspended in the incubation medium $\left(118 \mathrm{mmol} / \mathrm{l} \mathrm{NaCl}, 5.0 \mathrm{mmol} / \mathrm{l} \mathrm{KCl}, 1.2 \mathrm{mmol} / 1 \mathrm{KH}_{2} \mathrm{PO}_{4}, 1.2 \mathrm{mmol} / \mathrm{l}\right.$ $\mathrm{MgSO}_{4}, 25 \mathrm{mmol} / / \mathrm{NaHCO}_{3}, 2.5 \mathrm{mmol} / / \mathrm{CaCl}_{2}, 13 \mathrm{mmol} / \mathrm{l} \mathrm{HEPES}$, and $10.0 \mathrm{mmol} / \mathrm{l}$ glucose) to yield a cell density of $1.6 \times 10^{6}$ viable cells $/ \mathrm{ml}$ for the uptake study. The number of viable cells was determined using trypan blue staining. Before the uptake studies, the cell suspension-and test solution-containing test substrates were prewarmed in an incubator at $37^{\circ} \mathrm{C}$ for 5 minutes. The uptake studies were initiated by the addition to the cell suspension of an equal volume of buffer containing $\left[{ }^{14} \mathrm{C}\right]$ fasiglifam $(0.3 \mu \mathrm{mol} / \mathrm{l})$ or $\left[{ }^{3} \mathrm{H}\right]$ pravastatin $(0.3 \mu \mathrm{mol} / \mathrm{l})$, a substrate of organic anion transporting polypeptides (Oatps), in the presence or absence of $10 \mu \mathrm{mol} / \mathrm{l}$ cyclosporin A, a potent inhibitor of Oatps and sodium 
taurocholate cotransporting polypeptide (final density of the hepatocytes: $0.8 \times 10^{6} \mathrm{cell} / \mathrm{ml}$ as viable cells). After incubation at $37^{\circ} \mathrm{C}$ or $4^{\circ} \mathrm{C}$ for designated times, reaction solutions were gently added to the cell separation tubes. The tubes were immediately centrifuged at $10,000 \mathrm{~g}$ for 15 seconds at room temperature. The reaction was terminated by transferring the cells to a potassium hydroxide layer, and then an aliquot of the remaining reaction solution was measured for radioactivity using a LSC. The uptake clearances (CLs) of pravastatin were estimated from the uptake volumes at $37^{\circ} \mathrm{C}$ for 20 and 90 seconds. The uptake CLs of fasiglifam were estimated from the uptake volumes of fasiglifam at $37^{\circ} \mathrm{C}$ for 30 seconds and at $4^{\circ} \mathrm{C}$ for 20 and 60 seconds, because the uptake CL of fasiglifam was too high to evaluate at $37^{\circ} \mathrm{C}$ between two time points. All incubations were made in duplicate.

Inhibitory Effects of Fasiglifam and Fasiglifam-G on Hepatobiliary Transporters. Transport studies were performed using the rapid filtration technique (van Staden et al., 2012). Briefly, $40 \mu \mathrm{l}$ of transport medium $[50 \mathrm{mmol} / 1$ 3-morpholinopropanesulfonic acid-Tris, $70 \mathrm{mmol} / \mathrm{l} \mathrm{KCl}$, and $7.5 \mathrm{mmol} / \mathrm{l} \mathrm{MgCl}_{2}$ (pH 7.0) for Mrp2, or $10 \mathrm{mmol} / \mathrm{l}$ HEPES-Tris, $100 \mathrm{mmol} / 1 \mathrm{KNO}_{3}, 10 \mathrm{mmol} / \mathrm{l}$ $\mathrm{Mg}\left(\mathrm{NO}_{3}\right)_{2}$, and $50 \mathrm{mmol} / \mathrm{l}$ sucrose ( $\mathrm{pH}$ 7.4) for Bsep] containing radiolabeled compounds $\left(\left[{ }^{3} \mathrm{H}\right] \mathrm{E}_{2} 17 \beta \mathrm{G}\right.$ for Mrp2 and $\left[{ }^{3} \mathrm{H}\right] \mathrm{TCA}$ for Bsep) was preincubated at $37^{\circ} \mathrm{C}$ for 5 minutes. The transport reaction was initiated by the addition of $10 \mu \mathrm{l}$ of membrane vesicle suspension ( $50 \mu \mathrm{g}$ of protein) into the preincubated transport medium. The reaction mixture contained $5 \mathrm{mmol} / \mathrm{l}$ of ATP or AMP. The transport reaction was terminated by addition of $1 \mathrm{ml}$ of ice-cold buffer $[40 \mathrm{mmol} / 1$ 3-morpholinopropanesulfonic acid-Tris and $70 \mathrm{mmol} / \mathrm{l} \mathrm{KCl}(\mathrm{pH}$ 7.0) for Mrp2, or $10 \mathrm{mmol} / \mathrm{l} \mathrm{HEPES}$-Tris, $100 \mathrm{mmol} / 1 \mathrm{KNO}_{3}$, and $50 \mathrm{mmol} / \mathrm{l}$ sucrose $(\mathrm{pH} 7.4$ ) for Bsep]. The stopped reaction mixture was filtered through a glass fiber filter (Merck Millipore, Darmstadt, Germany) and then washed twice with $10 \mathrm{ml}$ of icecold buffer. The radioactivity retained on the filter was determined using the LSC.

Because the initial linear uptake was confirmed for TCA into Bsep-expressing vesicles and $\mathrm{E}_{2} 17 \beta \mathrm{G}$ into Mrp2-expressing vesicles at 1 and 2 minutes, respectively, the uptake study for $\left[{ }^{3} \mathrm{H}\right] \mathrm{TCA}(0.2 \mu \mathrm{mol} / 1)$ and $\left[{ }^{3} \mathrm{H}\right] \mathrm{E}_{2} 17 \beta \mathrm{G}(0.02 \mu \mathrm{mol} / \mathrm{l})$ was conducted at 1 and 2 minutes, respectively, to estimate the $\mathrm{IC}_{50}$ values of fasiglifam and fasiglifam-G. The uptake CLs of $\left[{ }^{3} \mathrm{H}\right] \mathrm{TCA}$ or $\left[{ }^{3} \mathrm{H}\right] \mathrm{E}_{2} 17 \beta \mathrm{G}$ into the membrane vesicles were investigated in the presence of $1,3,10,30$, and $100 \mu \mathrm{mol} / 1$ of fasiglifam or fasiglifam-G. All incubations were made in triplicate. The $\mathrm{IC}_{50}$ values were obtained from curve fitting of the concentration-inhibition curves by nonlinear regression analysis by using SAS System version 8.02 (SAS Institute Inc., Cary, NC).

\section{Measurement of Radioactivity}

Radioactivity in the plasma, bile, in vitro biologic samples, and organic solvent extracts was measured directly using the LSC [LSC-5100 (Aloka Co., Ltd., Tokyo, Japan); Tri-Carb 2100TR, 2700TR, 2900TR, 3100TR, or 3110TR (PerkinElmer Inc.); or LS 6000IC or 6500 (Beckman Coulter, Inc., Brea, CA)] with HionicFluor (PerkinElmer Inc.) and liquid scintillator A (toluene-based scintillator; Wako Pure Chemical Industries Ltd.). Radioactivity in the liver homogenate was measured using the combustion method by employing a sample oxidizer (model 307 or A030701; PerkinElmer Inc.). Resultant $\left[{ }^{14} \mathrm{C}_{\mathrm{CO}_{2}}\right.$ was trapped in carbon dioxide absorbent (Carbo-Sorb E; PerkinElmer Inc.), and Permafluor $\mathrm{E}^{+}$(PerkinElmer Inc.) or liquid scintillator B (toluene-based scintillator; Wako Pure Chemical Industries Ltd.) was added, and then radioactivity was assayed using the LSC. Metabolite profiles in the dog liver samples obtained after repeat dosing were generated using HPLC with fraction collection, followed by measuring the radioactivity employing an ultra-low-background LSC (1220 Quantulus; PerkinElmer Inc.) by using Ultima Gold uLLT (PerkinElmer Inc.) as a liquid scintillator. In the online detection during HPLC, radioactivity was measured using an online radioisotope detector (model 505TR or 625TR; PerkinElmer Inc.), with Ultima-Flo AP (PerkinElmer Inc.) used as the liquid scintillator. The lower limit of quantitation was defined as twice and three times the background value for offline and online detection, respectively.

\section{Quantification of Fasiglifam in Plasma}

The concentrations of fasiglifam in plasma in the dog multiple dosing study were determined using HPLC. Forty-microliter aliquots of the plasma samples were mixed with $960 \mu \mathrm{l}$ of the $5 \%$ ammonia solution. The mixed samples were loaded onto solid-phase extraction cartridges (Oasis MAX extraction cartridge, $1 \mathrm{cc} / 30 \mathrm{mg}$; Waters Corporation, Milford, MA), which were successively washed with $1 \mathrm{ml}$ of the $5 \%$ ammonia solution and $1 \mathrm{ml}$ of methanol. The analytes were eluted to glass test tubes with $1 \mathrm{ml}$ of the $1 \%$ trifluoroacetic acid methanol solution. The eluates were mixed with $100 \mu \mathrm{l}$ of the propylene glycol solution. These mixtures were evaporated under a nitrogen stream at $40^{\circ} \mathrm{C}$. The residues were dissolved in $300 \mu \mathrm{l}$ of the mobile phase by vortex mixing for about 30 seconds, sonicating for about 1 minute, and vortex mixing for about 30 seconds. The mixtures were centrifuged at approximately $1800 \mathrm{~g}$ at room temperature for 3 minutes. The supernatants were injected into the Shimadzu LC-VP HPLC system (Shimadzu Corporation) equipped with an XBridge C18 column ( $4.6 \mathrm{~mm}$ i.d. $\times 150 \mathrm{~mm}, 5 \mu \mathrm{m}$; Waters Corporation) maintained at $40^{\circ} \mathrm{C}$. The mobile phase consisted of $10 \mathrm{mmol} / \mathrm{l}$ ammonium formate $(\mathrm{pH} 3) /$ acetonitrile $(9: 11, \mathrm{v} / \mathrm{v})$. A flow rate of $1 \mathrm{ml} / \mathrm{min}$ was maintained for the entire run. The eluted fasiglifam was monitored with absorbance at $235 \mathrm{~nm}$. The lower limit of quantitation for fasiglifam was $0.2 \mu \mathrm{g} / \mathrm{ml}$.

\section{Solubility of Fasiglifam and Fasiglifam-G in Bile}

The solubility of fasiglifam and fasiglifam-G in dog and human bile was assessed by measuring the recovery of radioactivity in the supernatant of the bile after centrifugation of the bile samples spiked with $\left[{ }^{14} \mathrm{C}\right]$ fasiglifam and $\left[{ }^{14} \mathrm{C}\right]$ fasiglifam-G at concentrations of 1,3 , and $10 \mathrm{mg} / \mathrm{ml}$. Dog bile was obtained from eight male and female beagle dogs with an age range of 10-12 months. Human bile was obtained from nine men and women with an age range of 55-82 years. The spiked samples were set in a water bath at $37^{\circ} \mathrm{C}$ for 60 minutes and centrifuged at approximately $1850 \mathrm{~g}$ at $25^{\circ} \mathrm{C}$ for 10 minutes, and then the radioactivity in the supernatant was measured as the recovery of radioactivity.

\section{Prediction Methods for Fasiglifam in Bile}

The maximum concentration of fasiglifam in bile $\left(C_{\max \text { bile }}\right)$ was estimated using the maximum plasma concentration $\left(C_{\text {max } \_ \text {plasma }}\right)$ of fasiglifam, biliary clearance $\left(\mathrm{CL}_{\mathrm{b}}\right)$, and the bile flow rate (eq. 1):

$$
C_{\text {max_bile }}=\frac{\mathrm{CL}_{\mathrm{b}} \times C_{\text {max_plasma }}}{\text { Bile flow rate }}
$$

Crystal formation is likely to be driven by $C_{\text {max_bile }}$ of fasiglifam and fasiglifam-G. The steady-state $C_{\text {max_plasma }}$ of fasiglifam can be used to predict the corresponding value at steady state in bile (eq. 1). The biliary clearance of fasiglifam may be determined using the biliary excretion ratio (ER) and the total plasma clearance $\left(\mathrm{CL}_{\mathrm{tot}}\right)$ values of fasiglifam $\left(\mathrm{CL}_{\mathrm{b}}=\mathrm{ER} \times \mathrm{CL}_{\mathrm{tot}}\right)$.

\section{Data Analysis}

Data are expressed as mean or mean \pm S.D. values of the results from two to four animals. Values for $C_{\text {max_plasma }}$ and time to $C_{\text {max }}$ plasma (time to maximum concentration) were directly noted from the data. The terminal half-life and area under the plasma versus time-concentration curve were calculated from the measured values by using the noncompartmental model in WinNonlin (version 4.1; Pharsight Corporation, Mountain View, CA).

\section{Results}

\section{MALDI TOF MS}

Direct mass spectrometric measurements were conducted for lesion sites containing the foreign body material in the liver sections from a dog in a 39-week oral gavage toxicity study of fasiglifam. The ions that corresponded to fasiglifam and fasiglifam- $G$ were detected in the lesions containing the foreign body material (Supplemental Fig. 3), whereas the corresponding peaks were not observed in normal regions (Supplemental Fig. 4). The MS/MS experiment was performed at $\mathrm{m} / \mathrm{z}, 563.2$ and 739.2 as precursor ions. Therefore, the ion at $\mathrm{m} / \mathrm{z} 563.2$ was identified to be potassiated fasiglifam by comparing it with the MS/MS spectrum of the reference standard of fasiglifam (Supplemental Fig. 5). The ion at $\mathrm{m} / \mathrm{z} 739.2$ was estimated to be fasiglifam-G because a typical neutral loss of 176 Da was observed in the MS/MS spectrum (Supplemental Fig. 6). The predominant product ion in this MS/MS experiment also corresponded to the mass value of potassiated fasiglifam $(\mathrm{m} / \mathrm{z}, 563.2)$. The relative intensities of the observed ions corresponding to both fasiglifam and 
fasiglifam-G were much higher in the spots in the lesions containing the foreign body materials than in those in the normal regions (Supplemental Table 2).

\section{Evaluation of the Metabolic Profiles of $\left[{ }^{14} \mathrm{C}\right]$ Fasiglifam in the Plasma and Liver Samples}

The concentrations of radioactivity in the plasma and liver were determined in rats and dogs treated with $\left[{ }^{14} \mathrm{C}\right]$ fasiglifam at single oral doses of 2 and $200 \mathrm{mg} / \mathrm{kg}$. Following a single oral administration of the same dose, the concentrations of total radioactivity in the rat liver were 2- to 6-fold higher than those in the dog liver (Table 1). Metabolic profiling studies indicated that fasiglifam was the major component in the plasma ( $>85 \%$ of radioactivity) and liver ( $>57 \%$ of radioactivity) in rats and dogs. The concentrations of fasiglifam in the rat liver were 2- to 10-fold higher than those in the dog liver, while the concentrations of fasiglifam- $G$ in the liver tended to be higher in dogs than in rats at the same oral dose (Fig. 2). The concentrations of fasiglifam-G were significantly lower than those of fasiglifam in the livers of rats and dogs.

\section{Evaluation of the Potential Covalent Binding of Fasiglifam-G to Plasma and Liver Proteins}

The potential covalent binding of fasiglifam-G (an acyl glucuronide) to the plasma and liver proteins was evaluated in rats and dogs treated with $\left[{ }^{14} \mathrm{C}\right]$ fasiglifam. Following a single oral and/or intravenous administration of $\left[{ }^{14} \mathrm{C}\right]$ fasiglifam to intact rats and dogs, the mean total recovery of radioactivity ranged from $95.9 \%$ to $99.3 \%$ (Kogame et al., 2018). These findings indicate that the administered $\left[{ }^{14} \mathrm{C}\right]$ fasiglifam was completely excreted in both species. No acyl migration of $\left[{ }^{14} \mathrm{C}\right]$ fasiglifam- $\mathrm{G}$ was detected in the plasma and liver of rats and dogs after oral administration of $\left[{ }^{14} \mathrm{C}\right]$ fasiglifam, and the total radioactivity in the plasma and liver of rats and dogs was extracted completely at an efficiency ranging from $91 \%$ to $109 \%$. In addition, potential covalent binding of fasiglifam and its metabolites to the microsome samples was evaluated. In vitro cytochrome P450 and uridine diphosphate glucuronosyltransferase reaction phenotyping studies generated oxidative metabolites (2',6'-dimethyl-4'-[3-(methylsulfonyl)propoxy]biphenyl-3carboxylic acid, T-1676427, and unidentified metabolites) and fasiglifam-G, respectively (Kogame et al., 2018). The recovery of the radioactivity through the pretreatment ranged from $98.4 \%$ to $104.9 \%$.

\section{Evaluation of $\left[{ }^{14} \mathrm{C}\right]$ Fasiglifam Accumulation in the Plasma and Liver of Dogs}

Fourteen-day repeat-dose studies were conducted in male dogs at an oral $\left[{ }^{14} \mathrm{C}\right]$ fasiglifam dose of $200 \mathrm{mg} / \mathrm{kg}$ per day. The concentration-time

\section{TABLE 1}

Concentrations of radioactivity in the plasma and liver after administration of a single oral dose of $\left[{ }^{14} \mathrm{C}\right]$ fasiglifam in rats and $\operatorname{dogs}$

Data were obtained from one animal for each collection time.

\begin{tabular}{lrrrr}
\hline \multirow{2}{*}{ Species } & Dose & Time & \multicolumn{2}{c}{ Concentration of Radioactivity $(\mu \mathrm{g} \mathrm{Eq} / \mathrm{ml}$ or g) } \\
\cline { 4 - 5 } & & & Plasma & Liver \\
\hline \multirow{3}{*}{ Rat } & $m g / k g$ & $h$ & & \\
& 2 & 1 & 4.98 & 18.1 \\
& 2 & 8 & 3.32 & 13.8 \\
\multirow{4}{*}{ Dog } & 200 & 1 & 237 & 275 \\
& 200 & 8 & 352 & 341 \\
& 2 & 2 & 5.04 & 10.6 \\
& 200 & 2 & 1.84 & 179 \\
& 200 & 8 & 315 & 55.3 \\
\hline
\end{tabular}

profiles of total radioactivity and fasiglifam after the 14th administration were similar to those after the first, seventh, and 13th administrations of $\left[{ }^{14} \mathrm{C}\right]$ fasiglifam (Supplemental Fig. 7), suggesting that neither total radioactivity nor fasiglifam accumulated in the plasma during the 14-day dosing period.

In the liver, the concentrations of total radioactivity, fasiglifam, and fasiglifam-G at 2 and 8 hours postdose of the 14th administration were similar or slightly higher than those after the first administration (Supplemental Table 3), indicating that there was no appreciable accumulation of total radioactivity, fasiglifam, or fasiglifam-G in the liver after 14 days of repeated dosing.

\section{Evaluation of the Disposition and Metabolism of $\left[{ }^{14} \mathrm{C}\right]$ Fasiglifam in Rats and Dogs}

Disposition and metabolism of $\left[{ }^{14} \mathrm{C}\right]$ fasiglifam was evaluated in BDC male rats and in dogs following a single intraduodenal dose in rats and after oral administration to dogs. At a dose of $2 \mathrm{mg} / \mathrm{kg}$, the mean total recovery concentrations of radioactivity at 24 hours postdose were $84.5 \%$ and $82.4 \%$ in rats and dogs, respectively; $81.4 \%$ and $78.2 \%$ of the dosed radioactivity were excreted into the bile of rats and dogs, respectively. These results showed that $\left[{ }^{14} \mathrm{C}\right]$ fasiglifam was well absorbed in rats and dogs at a dose of $2 \mathrm{mg} / \mathrm{kg}$, and that the absorbed fasiglifam and related compounds were mainly excreted into the bile. At a dose of $200 \mathrm{mg} / \mathrm{kg}$, the mean total recovery concentrations of radioactivity at 24 hours postdose were $84.4 \%$ and $54.7 \%$ in rats and dogs, respectively, with $54.1 \%$ and $42.0 \%$ of the dosed radioactivity being excreted into the bile for both, respectively. Data regarding excretion of total radioactivity are presented in Supplemental Table 4. A previous study showed that metabolism plays a greater role in the elimination of fasiglifam than biliary and urinary excretion of fasiglifam in both species, and glucuronidation of fasiglifam was the predominant metabolic pathway in both species (Kogame et al., 2018). The proposed metabolic pathways of fasiglifam are presented in Supplemental Fig. 8. Hence, fasiglifam-G was the main component in rat and dog bile (ranging from $41.6 \%$ to $78.8 \%$ of the total radioactivity), and metabolic profiles of bile samples were qualitatively similar between rats and dogs (Table 2). The biliary excretion of unchanged parent drug in dogs was similar to or slightly higher than that in rats (the adjusted biliary excretion ratios of fasiglifam were $13.8 \%$ and $7.0 \%$ of the dose in dogs and rats, respectively, at $2 \mathrm{mg} / \mathrm{kg}$ ), whereas the amount of fasiglifam-tau formed in dogs was at least 3-fold lower than that in rats.

\section{Hepatic Transporter Studies of Fasiglifam and Fasiglifam-G}

The uptake of $\left[{ }^{14} \mathrm{C}\right]$ fasiglifam into hepatocytes was investigated in rats and $\operatorname{dog} s$, and the results are summarized in Table 3. The hepatic uptake of $\left[{ }^{3} \mathrm{H}\right]$ pravastatin in hepatocytes was also investigated as a positive control for evaluating Oatp function. In the presence of cyclosporin A, the hepatic uptake CL of pravastatin was significantly reduced in rat and dog hepatocytes. These findings are consistent with reported data (Shitara et al., 2004; Wilby et al., 2011), suggesting that the rat and dog hepatocytes used in the present study retained Oatp function. The hepatic uptake $\mathrm{CL}$ values of $\left[{ }^{14} \mathrm{C}\right]$ fasiglifam were comparable between rats and dogs, indicating that no species difference existed in the hepatic uptake CL of $\left[{ }^{14} \mathrm{C}\right]$ fasiglifam. The uptake $\mathrm{CL}$ values of $\left[{ }^{14} \mathrm{C}\right]$ fasiglifam in rats and dogs were $\geq 43$-fold higher than those of $\left[{ }^{3} \mathrm{H}\right]$ pravastatin, a substrate for Oatps. In addition, cyclosporin $\mathrm{A}$, an inhibitor of Oatps and sodium taurocholate cotransporting polypeptide, had no or little inhibition $(<20 \%)$ on the hepatic uptake $\mathrm{CL}$ of $\left[{ }^{14} \mathrm{C}\right]$ fasiglifam in rats and dogs. These findings suggest that 
A

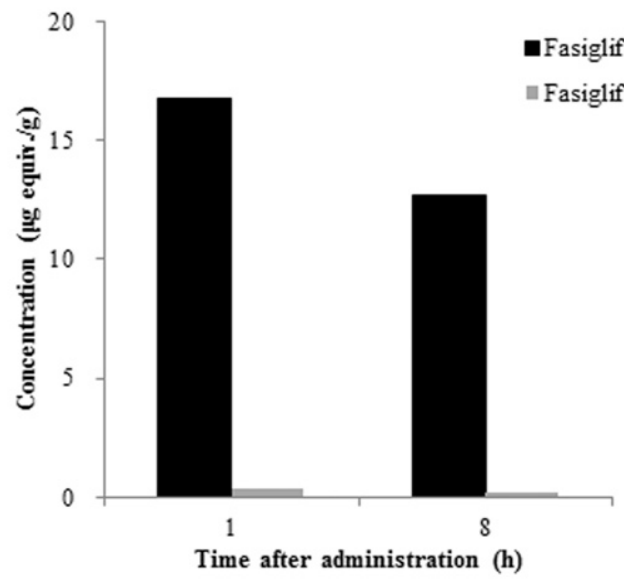

C

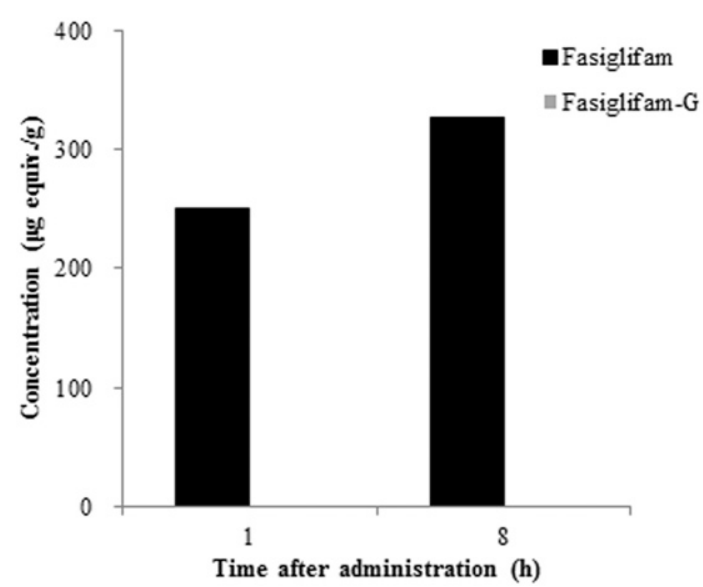

B

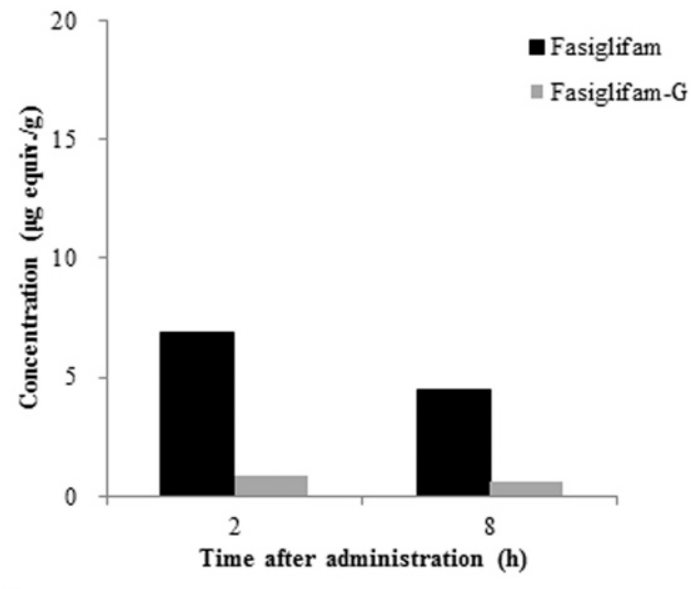

D

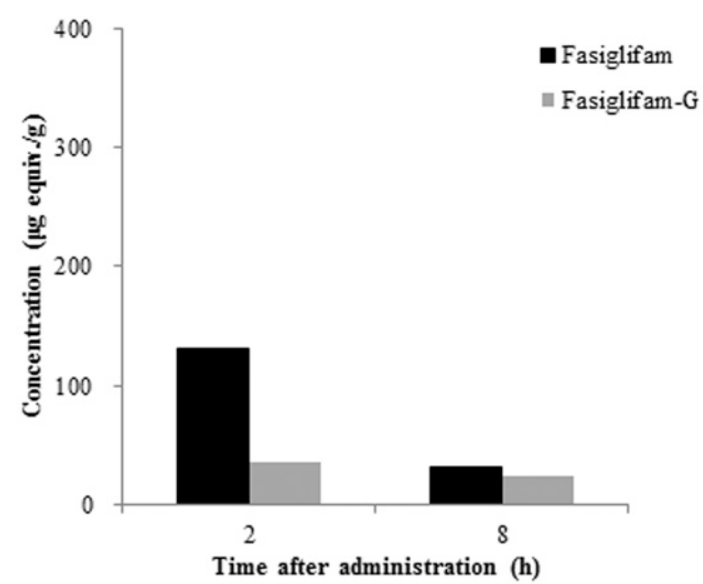

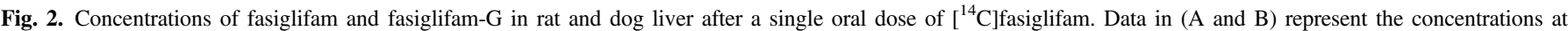

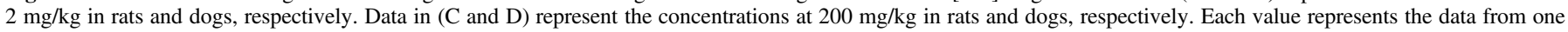
animal.

passive diffusion plays a more important role than transporter(s) in the hepatic uptake of $\left[{ }^{14} \mathrm{C}\right]$ fasiglifam in rats and dogs. The inhibitory effects of fasiglifam and fasiglifam- $\mathrm{G}$ on hepatobiliary transporters were evaluated, and the results are summarized in Table 4. No obvious species differences in potential inhibition were observed.

\section{TABLE 2}

Mean levels (percentages in parentheses) of fasiglifam and its metabolites in the bile of rats and dogs during $0-24 \mathrm{~h}$ following a single dose of $\left[{ }^{14} \mathrm{C}\right]$ fasiglifam

Each value represents an average of two animals. Numbers in parentheses represent proportions to the total radioactivity (\%). The individual measurements are presented in Supplemental Table 5.

\begin{tabular}{lccccc}
\hline \multirow{2}{*}{ Compound } & \multicolumn{4}{c}{ Percentage of Administered Dose } \\
\cline { 2 - 4 } & \multicolumn{2}{c}{ Dog } & \multicolumn{2}{c}{ Rat } \\
\cline { 2 - 5 } \cline { 5 - 6 } & $2 \mathrm{mg} / \mathrm{kg}$ & $200 \mathrm{mg} / \mathrm{kg}$ & & $2 \mathrm{mg} / \mathrm{kg}$ & $200 \mathrm{mg} / \mathrm{kg}$ \\
\hline Total radioactivity & $78.2(100.0)$ & $42.0(100.0)$ & $81.4(100.0)$ & $54.1(100.0)$ \\
Fasiglifam & $10.8(13.8)$ & $4.6(11.0)$ & $5.7(7.0)$ & $5.3(9.8)$ \\
M-I & $<$ LOQ (0.0) & $<$ LOQ (0.0) & $<$ LOQ (0.0) & $1.3(2.4)$ \\
Fasiglifam-G & $39.4(50.4)$ & $33.1(78.8)$ & $38.8(47.7)$ & $22.5(41.6)$ \\
Fasiglifam-Tau & $4.5(5.8)$ & $0.2(0.5)$ & & $15.2(18.7)$ & $12.2(22.6)$ \\
Others & $23.5(30.0)$ & $4.2(9.7)$ & & $21.9(26.6)$ & $13.0(23.6)$ \\
\hline
\end{tabular}

$<$ LOQ, below the lower limit of quantitation; M-I, 2',6'-dimethyl-4'-[3-(methylsulfonyl)propoxy]biphenyl-3-carboxylic acid.
Determination of the Concentrations of Total Radioactivity, Fasiglifam, and Fasiglifam-G in Bile after Single or Repeated Dosing of $\left[{ }^{14} \mathrm{C}\right] \mathrm{Fasiglifam}$

Concentrations of Total Radioactivity in the Bile. All bile samples described in this study were collected from the common bile duct of rats and dogs and/or from the gallbladder of dogs. Time-course studies showed that the concentrations of total radioactivity in dog bile were up to 7-fold higher than those in rat bile after receiving a single oral

\section{TABLE 3}

Uptake of $\left[{ }^{14} \mathrm{C}\right]$ fasiglifam and $\left[{ }^{3} \mathrm{H}\right]$ pravastatin into rat and dog hepatocytes and the inhibitory effect of cyclosporin A

\begin{tabular}{|c|c|c|c|c|c|}
\hline \multirow{3}{*}{ Compound } & \multirow{3}{*}{ Concentration } & \multicolumn{4}{|c|}{ Uptake Clearance ( $\mu \mathrm{l} / \mathrm{min}$ per miligram protein) } \\
\hline & & \multicolumn{2}{|c|}{ Rat } & \multicolumn{2}{|c|}{ Dog } \\
\hline & & Rs704 & RS740 & DB252 & DB268 \\
\hline & нmol/l & & & & \\
\hline Pravastatin & 0.3 & 15.8 & 19.9 & 4.8 & 2.7 \\
\hline Pravastatin + CyA & $0.3+10$ & 2.8 & 3.2 & 0.7 & 0.9 \\
\hline Fasiglifam & 0.3 & 689.6 & 858.6 & 586.8 & 522.6 \\
\hline Fasiglifam + CyA & $0.3+10$ & 632.8 & 715.2 & 556.2 & 421.8 \\
\hline
\end{tabular}

CyA, cyclosporin A. 
TABLE 4

The effect of $\mathrm{IC}_{50}$ values of fasiglifam and fasiglifam-G on hepatobiliary transporters

\begin{tabular}{|c|c|c|c|c|}
\hline \multirow{3}{*}{ Species } & \multicolumn{4}{|c|}{$\mathrm{IC}_{50}(\mu \mathrm{mol} / \mathrm{l})$} \\
\hline & \multicolumn{2}{|c|}{ Mrp2 } & \multicolumn{2}{|c|}{ Bsep } \\
\hline & Fasiglifam & Fasiglifam-G & Fasiglifam & Fasiglifam-G \\
\hline Rat & - & 2.9 & 20.6 & 82.5 \\
\hline Dog & - & 5.1 & 16.1 & 18.2 \\
\hline
\end{tabular}

-, not determined.

(dogs) or intraduodenal (rats) dose of 2 or $200 \mathrm{mg} / \mathrm{kg}$ (Fig. 3). The concentrations of total radioactivity in the bile were also determined in dogs after 14 days of repeated dosing at an oral $\left[{ }^{14} \mathrm{C}\right]$ fasiglifam dose of $200 \mathrm{mg} / \mathrm{kg}$ per day. After the 14th administration, the concentrations of total radioactivity in dog bile at 2 and 8 hours postdose were 12,143 and $18,357 \mu \mathrm{g} \mathrm{Eq} / \mathrm{ml}$, respectively, which were approximately similar to or slightly higher than those in dogs after a single oral dose of $200 \mathrm{mg} / \mathrm{kg}$ (6668 and 14,089 $\mu \mathrm{g} \mathrm{Eq} / \mathrm{ml}$ at 2 and 8 hours, respectively). The results also indicated that the concentrations of total radioactivity in dog bile were 54- to 173-fold higher than those in the liver after 14 days of repeated dosing at an oral dose of $200 \mathrm{mg} / \mathrm{kg}$ per day (224 and $106 \mu \mathrm{g} \mathrm{Eq} / \mathrm{ml}$ at 2 and 8 hours, respectively).

\section{A}

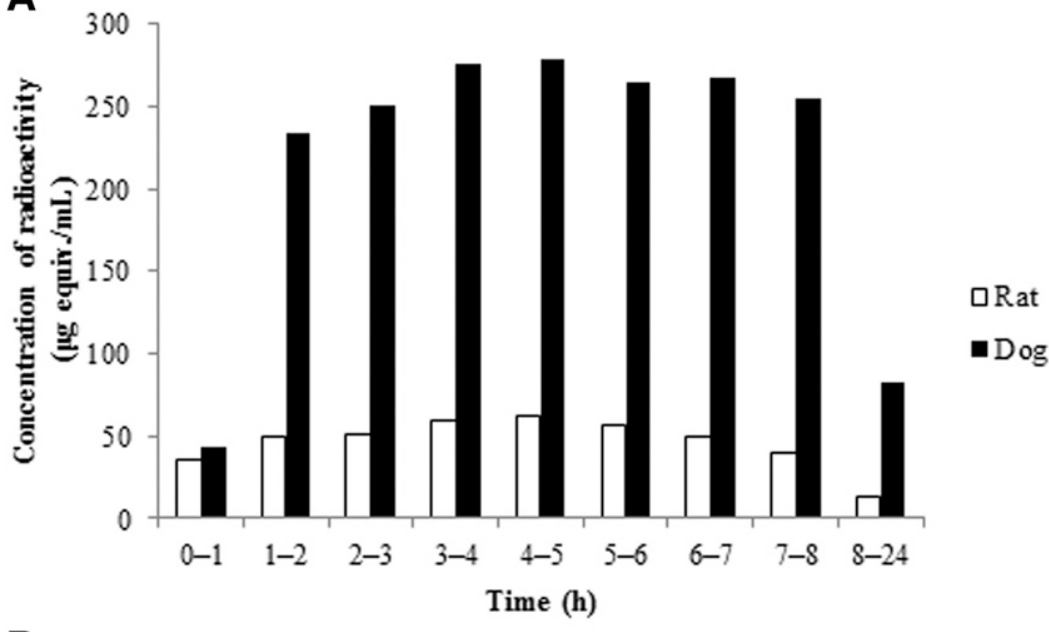

B

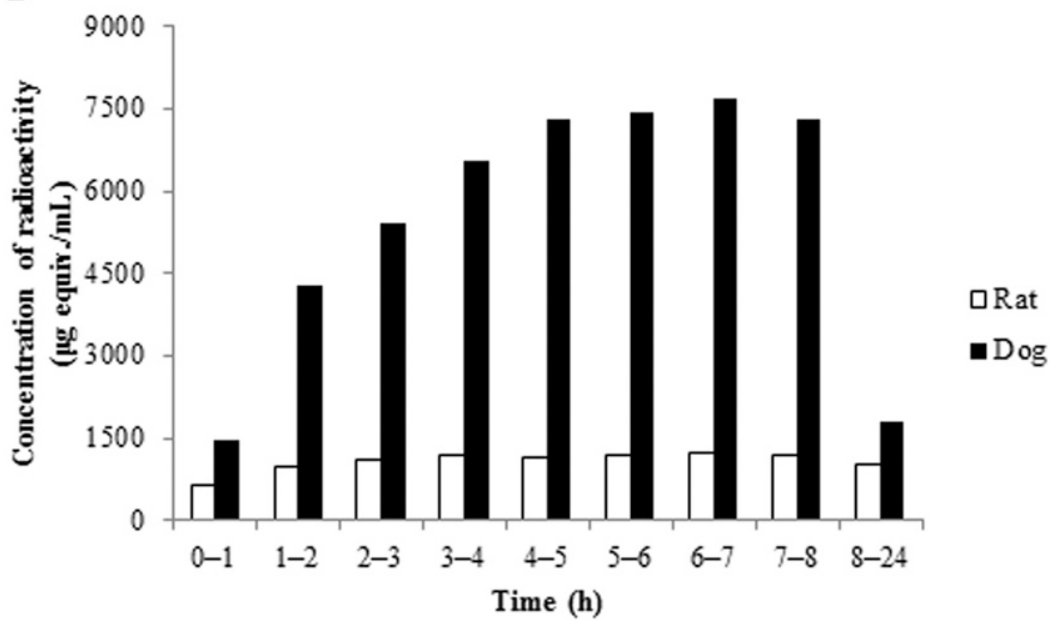

Concentrations of Fasiglifam and Fasiglifam-G in the Bile. The concentrations of fasiglifam and its metabolites in the pooled rat and dog bile over 24 hours after a single intraduodenal or oral dose of 2 or $200 \mathrm{mg} / \mathrm{kg}$ were determined. Within the same dose group, the concentrations of fasiglifam and fasiglifam-G in the dog bile were 5- to 10-fold higher than those in the rat bile (Table 5). The concentrations of 2', $6^{\prime}$-dimethyl4'-[3-(methylsulfonyl)propoxy]biphenyl-3-carboxylic acid and fasiglifamtau in dog bile were similar or lower than those in rat bile. The concentrations of fasiglifam and fasiglifam- $\mathrm{G}$ in dog bile were also determined after 14 days of repeated dosing of $\left[{ }^{14} \mathrm{C}\right]$ fasiglifam at $200 \mathrm{mg} / \mathrm{kg}$ per day. Following repeated dosing, the average concentrations of fasiglifam and fasiglifam-G in dog bile at 2 and 8 hours postdose ranged from 5280 to $11,087 \mu \mathrm{g} / \mathrm{ml}$ (individual values in four dogs ranged from 3509 to $12,956 \mu \mathrm{g} / \mathrm{ml}$ ) (Table 6). Note that since the measurements in Table 5 are concentrations over 24 hours, the values in Table 5 cannot be compared with the individual time measurements in Table 6 . No precipitate/crystalline material was observed in the bile collected from the gallbladder during the study. This study also indicated that the concentrations of fasiglifam and fasiglifam-G in the bile slightly increased ( $\leq 2$-fold) with repeated dosing compared with those in dogs after a single oral dose of $200 \mathrm{mg} / \mathrm{kg}$.

\section{Determination of Bile Flow Rate and $\mathrm{pH}$ in Rats and Dogs in the Presence of $\left[{ }^{14} \mathrm{C}\right]$ Fasiglifam}

Bile flow rates and $\mathrm{pH}$ values in rats and dogs were determined following a single intraduodenal (rats) or oral (dogs) administration of
Fig. 3. Time course of the concentrations of radioactivity in bile after the administration of a single dose of $\left.{ }^{14} \mathrm{C}\right]$ fasiglifam at $2 \mathrm{mg} / \mathrm{kg}$ (A) and $200 \mathrm{mg} / \mathrm{kg}$ (B). Each value represents an average of two animals. The individual measurements are presented in Supplemental Table 6. 
TABLE 5

Concentrations of fasiglifam and its metabolites in pooled bile over $24 \mathrm{~h}$ after a single dose of $\left[{ }^{14} \mathrm{C}\right]$ fasiglifam

$\left[{ }^{14} \mathrm{C}\right]$ fasiglifam was administered intraduodenally to rats and orally to dogs. The individual measurements are presented in Supplemental Table 7.

\begin{tabular}{clccc}
\hline \multirow{2}{*}{ Dose } & Compound & \multicolumn{2}{c}{ Concentration $(n=2)$} & \multirow{2}{*}{ Dog/Rat Fold Difference } \\
\cline { 3 - 4 } & & Rat & Dog & \\
\hline \multirow{2}{*}{$m g / k g$} & & $\mu g E q / m l$ & $\mu g E q / m l$ & \\
2 & Fasiglifam & 1.80 & 18.2 & 10.1 \\
& Fasiglifam-G & 12.4 & 66.6 & 5.4 \\
& Fasiglifam-tau & 4.83 & 7.65 & 1.6 \\
& M-I & $<$ LOQ & $<$ LOQ & NA \\
200 & Fasiglifam & 101 & 470 & 4.7 \\
& Fasiglifam-G & 433 & 3341 & 7.7 \\
& Fasiglifam-tau & 231 & 16.0 & $<1$ \\
& M-I & 23.9 & $<$ LOQ & $<1$ \\
\hline
\end{tabular}

$<$ LOQ, below the lower limit of quantitation; M-I, 2',6'-dimethyl-4'-[3-(methylsulfonyl)propoxy]biphenyl-3-carboxylic acid; NA, not available.

$\left[{ }^{14} \mathrm{C}\right]$ fasiglifam at 0,2 , or $200 \mathrm{mg} / \mathrm{kg}$ (where $0 \mathrm{mg} / \mathrm{kg}$ is the vehicle control). Data from the literature (Davies and Morris, 1993) and the present study indicate that the mean normal bile flow rate adjusted by body weight in dogs is about 4- to 8-fold slower than that in rats. At $2 \mathrm{mg} / \mathrm{kg}$, the bile flow rates in rats and dogs were similar to those observed in the vehicle control groups (Table 7). At $200 \mathrm{mg} / \mathrm{kg}$, the bile flow rates in rats and dogs were slightly higher than those observed in the vehicle control groups (Table 7). No significant change in the $\mathrm{pH}$ values of bile in rats and dogs was observed after a single intraduodenal (rats) and oral (dogs) dose of $\left[{ }^{14} \mathrm{C}\right]$ fasiglifam at 2 or $200 \mathrm{mg} / \mathrm{kg}$ (Table 7). However, following 14 days of repeated dosing at an oral dose of $200 \mathrm{mg} / \mathrm{kg}$ per day, the $\mathrm{pH}$ of the dog bile increased to a range of 6.8-7.6.

\section{The In Vitro Solubility of Fasiglifam and Fasiglifam-G}

The in vitro solubility of fasiglifam and fasiglifam-G in dog and human bile was determined using individual bile samples collected from the respective species spiked with different concentrations of $\left[{ }^{14} \mathrm{C}\right]$ fasiglifam or $\left[{ }^{14} \mathrm{C}\right]$ fasiglifam-G. Results of these studies showed that the solubility limit for both fasiglifam and fasiglifam-G was approximately $3000 \mu \mathrm{g} / \mathrm{ml}$ in dog bile and approximately $1000 \mu \mathrm{g} / \mathrm{ml}$ in human bile (Table 8 ). No apparent effect of either total bile acid concentrations or bile $\mathrm{pH}$ was noted on solubility.

\section{Predicted Maximum Concentrations of Fasiglifam in Dog Bile}

Using eq. 1, we would have predicted a maximum biliary concentration of $2590 \mu \mathrm{g} / \mathrm{ml}$. This was calculated using the maximum plasma concentration of $315 \mu \mathrm{g} / \mathrm{ml}$ reported in Table 1 , the total dog CL of

TABLE 6

Concentrations of fasiglifam and fasiglifam- $\mathrm{G}$ in dog bile after a single or repeating dose of $\left[{ }^{14} \mathrm{C}\right]$ fasiglifam administered at $200 \mathrm{mg} / \mathrm{kg}$ per day

Data were obtained from one animal for each collection time at the first dose. Each value at the 14th dose represents the mean of two animals (the individual measurements are presented in Supplemental Table 8).

\begin{tabular}{ccr}
\hline \multirow{2}{*}{ Time after dosage } & \multicolumn{2}{c}{ Concentration } \\
\cline { 2 - 3 } & Fasiglifam & Fasiglifam-G \\
\hline \multicolumn{1}{c}{$h$} & $\mu g E q / g$ & $\mu g E q / g$ \\
First dose & & \\
2 & 2407 & 3934 \\
8 & 5002 & 7862 \\
Fourteenth dose & & 6863 \\
2 & 5280 & 11,087 \\
8 & 7269 & \\
\hline
\end{tabular}

TABLE 7

Range of bile flow rates and $\mathrm{pH}$ values (triplicate samples) after a single dose of $\left[{ }^{14} \mathrm{C}\right]$ fasiglifam

$\left[{ }^{14} \mathrm{Clfasiglifam}\right.$ was administered intraduodenally to rats and orally to dogs. The $\mathrm{pH}$ of the bile was obtained from 0 to $24 \mathrm{~h}$ postdose in rats and at 2 and $8 \mathrm{~h}$ postdose in dogs. The published bile flow rate is $90 \mathrm{ml} /$ day per kilogram in rats and $12 \mathrm{ml} /$ day per kilogram in dogs (Davies and Morris, 1993).

\begin{tabular}{lllllll}
\hline \multirow{2}{*}{ Species } & \multicolumn{2}{c}{ Bile Flow Rate $(\mathrm{ml} /$ day per kilogram $)(n=2)$} & & \multicolumn{2}{c}{$\mathrm{pH}(n=1)$} \\
\cline { 2 - 3 } \cline { 7 - 8 } & Vehicle & $2 \mathrm{mg} / \mathrm{kg}$ & $200 \mathrm{mg} / \mathrm{kg}$ & & $2 \mathrm{mg} / \mathrm{kg}$ & $200 \mathrm{mg} / \mathrm{kg}$ \\
\hline Rat & $44-49$ & $61-68$ & $92-115$ & & $7.4-7.7$ & $7.4-8.0$ \\
Dog & $12-13$ & 12 & $20-24$ & & $6.5-6.8$ & 6.8 \\
\hline
\end{tabular}

fasiglifam of $29.8 \mathrm{ml} / \mathrm{h}$ per kilogram reported by Kogame et al. (2018), the extraction ratio of 0.138 determined in Table 2 , and a bile flow rate of $0.5 \mathrm{ml} / \mathrm{h}$ per kilogram as given in Table 7 , which is also the value reported by Davies and Morris (1993).

\section{Discussion}

Fasiglifam treatment in 4- to 39-week repeated-dose toxicology studies in dogs resulted in liver toxicity characterized by the elevation of aspartate aminotransferase, ALT, alkaline phosphatase, and/or bilirubin. Associated histopathological changes were characterized as portal/periportal granulomatous inflammation with crystal formation. This toxicity was both dose and duration dependent. In the 39-week dog study, the liver toxicity observed at $150 \mathrm{mg} / \mathrm{kg}$ per day had either completely resolved or showed signs of recovery following a 13-week recovery period. The observed fasiglifam-related dog liver toxicity was characterized by conducting nonclinical mechanistic studies in vitro and in vivo. For comparison, mechanistic studies were also conducted in rats. In the repeated-dose toxicology studies in rats, the histopathological changes observed in dogs were not found in rats at doses up to $2000 \mathrm{mg} / \mathrm{kg}$ per day. For the in vivo mechanistic studies, oral doses of 2 and $200 \mathrm{mg} / \mathrm{kg}$ per day were used because $2 \mathrm{mg} / \mathrm{kg}$ per day was considered nontoxic (the human equivalent dose is approximately $67 \mathrm{mg} /$ day based on body surface area), whereas doses of $\geq 150 \mathrm{mg} / \mathrm{kg}$ per day (the human equivalent dose for $150 \mathrm{mg} / \mathrm{kg}$ per day in dog is $5000 \mathrm{mg} /$ day) resulted in liver toxicity after repeated dosing for $\geq 13$ weeks in dogs. MALDI TOF MS analysis indicated that the crystalline material in the liver sections of affected dogs contained fasiglifam and fasiglifam- $G$, indicating that the dog liver toxicity could be characterized in terms of pharmacokinetics and physicochemistry of fasiglifam and its related compounds.

No dog-specific metabolites were found in the plasma, liver, or bile; furthermore, no evidence of covalent binding of fasiglifam-G (an acyl glucuronide) to the plasma or liver proteins was noted in the dogs. Moreover, no significant accumulation of total radioactivity, fasiglifam, and/or fasiglifam-G was noted in the dog plasma or liver following 14 days of repeated oral dosing of $\left[{ }^{14} \mathrm{C}\right]$ fasiglifam at $200 \mathrm{mg} / \mathrm{kg}$ per day. Although the concentrations of fasiglifam in the rat liver were 2- to 10 -fold higher than those in the dog liver at the same dose level (Fig. 2), no histopathological liver injury was observed in any rat toxicology studies, suggesting that fasiglifam is not directly toxic to hepatocytes.

Transporter studies showed that passive diffusion played a more important role than active transport in the hepatic uptake of fasiglifam in rats and dogs. No species difference was noted between rats and dogs in the CL of fasiglifam hepatic uptake and fasiglifam-G hepatobiliary efflux, or potential inhibition of hepatobiliary transporters by fasiglifam and fasiglifam-G. Therefore, the involvement of hepatic transporters could not solely account for the differences in the liver findings in the rat and dog toxicity studies. 
TABLE 8

The in vitro solubility of $\left[{ }^{14} \mathrm{C}\right]$ fasiglifam and $\left[{ }^{14} \mathrm{C}\right]$ fasiglifam- $\mathrm{G}, \mathrm{pH}$, and total bile acid concentrations in dog bile

Values represent the range for eight dogs and nine humans.

\begin{tabular}{lccccc}
\hline \multirow{2}{*}{ Parameter } & \multicolumn{2}{c}{ Dog $(n=8)$} & & \multicolumn{2}{c}{ Human $(n=9)$} \\
\cline { 2 - 3 } & {$\left[{ }^{14} \mathrm{C}\right]$ Fasiglifam } & {$\left[{ }^{14} \mathrm{C}\right]$ Fasiglifam-G } & & {$\left[{ }^{14} \mathrm{C}\right]$ Fasiglifam } & {$\left[{ }^{14} \mathrm{C}\right]$ Fasiglifam-G } \\
\hline Recovery $^{a}$ at $1000 \mu \mathrm{g} / \mathrm{ml}(\%)$ & $91.6-96.7$ & $98.5-102$ & & $92.9-99.9$ & $91.7-98.0$ \\
Recovery $^{a}$ at $3000 \mu \mathrm{g} / \mathrm{ml}(\%)$ & $80.5-90.6$ & $92.0-103$ & & $63.9-96.4$ & $60.3-96.1$ \\
Recovery $^{a}$ at $10,000 \mu \mathrm{g} / \mathrm{ml}(\%)$ & $63.3-77.5$ & $55.0-102$ & & $20.6-78.1$ & $24.3-80.2$ \\
pH & \multicolumn{2}{c}{$6.8-7.8$} & & $6.4-7.6$ \\
Total bile acid concentration (mmol/l) & \multicolumn{2}{c}{$119-187$} & & $11.6-139$ \\
\hline
\end{tabular}

${ }^{a}$ Recovery of the spiked radioactive material.

Following a single intraduodenal or oral dose of $\left[{ }^{14} \mathrm{C}\right]$ fasiglifam at 2 or $200 \mathrm{mg} / \mathrm{kg}$ to rats and dogs, within the same dose group, the concentrations of total radioactivity, fasiglifam, and fasiglifam- $\mathrm{G}$ in dog bile were 5- to 10 -fold higher than those in rat bile (Table 5), whereas their excretion (percentage of administered dose) was comparable between the two species (Table 2). The differences in concentrations are attributed to the difference in bile flow rate between the two species. In the vehicle group, the bile flow rate in dogs (12 to $13 \mathrm{ml} /$ day per kilogram) was considerably slower than that in rats (44-49 $\mathrm{ml} /$ day per kilogram), which was consistent with published data (Davies and Morris, 1993). We note that the maximum plasma concentration reported here (Table 1) is consistent with that in the 39-week toxicity study in dogs, where a maximum concentration was $78 \mu \mathrm{g} / \mathrm{ml}$ for $40 \mathrm{mg} / \mathrm{kg}$ per day.

The bile concentration of fasiglifam was $7269 \mu \mathrm{g} / \mathrm{ml}$ at 8 hours after 14 days of repeated dosing of $\left[{ }^{14} \mathrm{C}\right]$ fasiglifam at $200 \mathrm{mg} / \mathrm{kg}$ per day, as shown in Table 6. This value is just under three times the calculated maximum biliary concentration in dogs of $2590 \mu \mathrm{g} / \mathrm{ml}$ (using eq. 1). We attempted to predict the maximum bile concentration in dogs using eq. 1 , to see if it would be possible to predict the maximum bile concentration in humans. Assuming that the extraction ratio is similar between dogs and humans (0.138), using the total human CL of fasiglifam of $14.6 \mathrm{ml} / \mathrm{h}$ per kilogram (Kogame et al., 2018), the maximum plasma concentration of $5.3 \mu \mathrm{g} / \mathrm{ml}$ observed in T2DM patients for a $50 \mathrm{mg}$ multiple daily dose study (Leifke et al., 2012), and a human bile flow rate of $0.24 \mathrm{ml} / \mathrm{h}$ per kilogram (Davies and Morris, 1993), a maximum bile concentration of $44.5 \mu \mathrm{g} / \mathrm{ml}$ would be predicted. We suggest that the solubility limit would be 20 -fold higher than the predicted fasiglifam concentration and that even if our calculations underpredict by one-third of the dog data, or even if the underprediction is 7-fold off in a worst case scenario, we would not expect biliary concentrations of fasiglifam to exceed the solubility limit for this compound. The in vitro solubility limit of fasiglifam in dog bile was approximately $3000 \mu \mathrm{g} / \mathrm{ml}$. However, no crystalline material was visually observed in the dog bile collected from the gallbladder at the end of the 14-day dosing period (data on file), possibly because it is difficult to find the microscopic nature of the crystals and/or the duration of the study was insufficient for crystal growing.

The $C_{\text {max bile }}$ of fasiglifam-G could not be estimated due to the low or undetectable levels of the metabolite in plasma. From Tables 2 and 5 it can be seen in both rats and dogs that the average fasiglifam-G bile concentrations over 24 hours range from 3.7- to 7.2-fold higher than the fasiglifam bile concentrations. The same pattern is observed for the dog concentrations at 2 and 8 hours in Table 6; however, the ratios are only 1.3-1.6 greater. If fasiglifam-G bile concentrations in humans follow the same relative pattern with fasiglifam as observed in rats and dogs, again we would not expect fasiglifam-G concentrations to exceed the solubility limits given in the previous human studies.

Histopathological examination of the liver from affected dogs in repeat-dose toxicity studies with fasiglifam showed that crystal formation occurred primarily in and around the portal triads of the dog liver, and granulomatous inflammation was a secondary reaction to the presence of crystals in these areas. Toxicity of this nature is dose dependent and explains the dose- and duration-dependent nature of the dog liver toxicity. In addition, assuming linear pharmacokinetics, we predicted the maximum concentrations of fasiglifam and fasiglifam- $\mathrm{G}$ in dog bile at the no observable adverse effect level (NOAEL) of $40 \mathrm{mg} / \mathrm{kg}$ per day. The predicted bile concentrations of these compounds at the NOAEL in the dog study did not exceed the solubility limit in the dog bile, indicating that crystal formation might not occur in the biliary tree of dogs at the NOAEL.

As shown previously, the predicted concentration in human bile indicated that the risk of crystal formation in human bile was minimal at the maximum recommended human dose $(50 \mathrm{mg})$. The safety margin based on the ratio of the dog area under the plasma versus time-concentration curve at the NOAEL $(854,000 \mathrm{ng} \cdot \mathrm{h} / \mathrm{ml}$ at $40 \mathrm{mg} / \mathrm{kg}$ ) (Wolenski et al., 2017) to the human area under the plasma versus time-concentration curve at the clinically efficacious dose $(61,463.4 \mathrm{ng} \cdot \mathrm{h} / \mathrm{ml}$ at $50 \mathrm{mg}$ ) (Mayer et al., 2014) was estimated to be more than 10-fold. However, the fasiglifam clinical development program was terminated owing to liver safety concerns. These facts indicate that the mechanism of liver toxicity might be different between humans and dogs. Although the fundamental pathogenesis of fasiglifaminduced liver injury in humans is still not well understood, nonclinical in vivo and in vitro studies have proposed theories for the pathogenesis caused by a fasiglifam-specific factor ( $\mathrm{Li}$ et al., 2015; Wolenski et al., 2017), in which the inhibitory effect of fasiglifam on several hepatobiliary transporters might provide a possible mechanism of fasiglifaminduced liver injury in human. Hepatobiliary transporters are known to play an important role in bile acid homeostasis (Köck and Brouwer, 2012; Chiang, 2013). Therefore, the inhibition of the transporters is considered to lead to abnormal intracellular concentration of bile acid, and subsequently liver injury since bile acids have a detergent-like property (Pauli-Magnus et al., 2005; Marion et al., 2007). However, in many cases, drug-induced liver injury cannot be predicted by nonclinical animal models (Chan and Benet, 2018), and since severe drug-induced liver injury has a very low incidence (see http://www.fda.gov/downloads/ Drugs/.../Guidances/UCM174090.pdf) (Björnsson et al., 2013; Fontana, 2014), in addition to drug-specific risk factors, patient-specific risk factors such as age, genetic polymorphisms, alcohol consumption, and/or medical history might also contribute to the susceptibility of drug-induced liver injury. Unfortunately, the patient-specific risk factors in fasiglifam clinical studies are not available.

In summary, high concentrations of fasiglifam and fasiglifam- $G$ that exceeded the solubility limits of these compounds in dog bile were most likely the main causes of dog liver toxicity observed in repeat-dose toxicology studies. The formation of crystals and toxicity development require high biliary concentrations and sufficient study duration. The present study showed the importance of pharmacokinetic studies in drug development in terms of clarifying the mechanism of toxicity and safety 
assessment, although the mechanism of fasiglifam-related liver toxicity in clinical studies is still unclear.

\section{Authorship Contributions}

Participated in research design: Kogame, Moriya, Pan, Fukui, Tagawa, Benet.

Conducted experiments: Kogame, Moriya, Mori, Ebihara.

Performed data analysis: Kogame, Moriya, Mori, Ebihara, Fukui, Tagawa.

Wrote or contributed to the writing of the manuscript: Kogame, Moriya,

Mori, Pan, Morohashi, Ebihara, Fukui, Tagawa, Benet.

\section{References}

Björnsson ES, Bergmann OM, Björnsson HK, Kvaran RB, and Olafsson S (2013) Incidence, presentation, and outcomes in patients with drug-induced liver injury in the general population of Iceland. Gastroenterology 144:1419-1425 [quiz e19-e20].

Chan R and Benet LZ (2018) Evaluation of the relevance of DILI predictive hypotheses in early drug development: review of in vitro methodologies vs BDDCS classification. Toxicol Res (Camb) 7:358-370

Chiang JY (2013) Bile acid metabolism and signaling. Compr Physiol 3:1191-1212.

Davies B and Morris T (1993) Physiological parameters in laboratory animals and humans. Pharm Res 10:1093-1095.

Fontana RJ (2014) Pathogenesis of idiosyncratic drug-induced liver injury and clinical perspectives. Gastroenterology 146:914-928.

Kaku K (2013) Fasiglifam as a new potential treatment option for patients with type 2 diabetes. Expert Opin Pharmacother 14:2591-2600.

Kaku K, Enya K, Nakaya R, Ohira T, and Matsuno R (2015) Efficacy and safety of fasiglifam (TAK-875), a G protein-coupled receptor 40 agonist, in Japanese patients with type 2 diabetes inadequately controlled by diet and exercise: a randomized, double-blind, placebo-controlled, phase III trial. Diabetes Obes Metab 17:675-681.

Kaku K, Enya K, Nakaya R, Ohira T, and Matsuno R (2016) Long-term safety and efficacy of fasiglifam (TAK-875), a G-protein-coupled receptor 40 agonist, as monotherapy and combination therapy in Japanese patients with type 2 diabetes: a 52-week open-label phase III study. Diabetes Obes Metab 18:925-929.

Köck K and Brouwer KL (2012) A perspective on efflux transport proteins in the liver. Clin Pharmacol Ther 92:599-612.

Kogame A, Lee R, Pan L, Sudo M, Nonaka M, Moriya Y, Higuchi T, and Tagawa Y (2018) Disposition and metabolism of the G protein-coupled receptor 40 agonist TAK-875 (fasiglifam) in rats, dogs, and humans. Xenobiotica 5:1-13.
Leifke E, Naik H, Wu J, Viswanathan P, Demanno D, Kipnes M, and Vakilynejad M (2012) A multiple-ascending-dose study to evaluate safety, pharmacokinetics, and pharmacodynamics of a novel GPR40 agonist, TAK-875, in subjects with type 2 diabetes. Clin Pharmacol Ther 92:29-39.

Li X, Zhong K, Guo Z, Zhong D, and Chen X (2015) Fasiglifam (TAK-875) inhibits hepatobiliary transporters: a possible factor contributing to fasiglifam-induced liver injury. Drug Metab Dispos 43:1751-1759.

Marcinak JF, Munsaka MS, Watkins PB, Ohira T, and Smith N (2018) Liver safety of fasiglifam (TAK-875) in patients with type 2 diabetes: review of the global clinical trial experience. Drug Saf 41:625-640.

Marion TL, Leslie EM, and Brouwer KL (2007) Use of sandwich-cultured hepatocytes to evaluate impaired bile acid transport as a mechanism of drug-induced hepatotoxicity. Mol Pharm 4:911-918.

Mayer M, Nudurupati S, Peng X, and Marcinak J (2014) Evaluation of the pharmacokinetics and safety of a single oral dose of fasiglifam in subjects with normal or varying degrees of impaired renal function. Drugs $R$ D 14:273-282.

Negoro N, Sasaki S, Mikami S, Ito M, Suzuki M, Tsujihata Y, Ito R, Harada A, Takeuchi K, Suzuki N, et al. (2010) Discovery of TAK-875: a potent, selective, and orally bioavailable GPR40 agonist. ACS Med Chem Lett 1:290-294.

Onishi A, Kogame A, Tagawa Y, Kondo T, and Asahi S (2013) Evaluation of low background liquid scintillation counter for non-clinical ADME studies. Xenobiotica 43:520-526.

Pauli-Magnus C, Stieger B, Meier Y, Kullak-Ublick GA, and Meier PJ (2005) Enterohepatic transport of bile salts and genetics of cholestasis. J Hepatol 43:342-357.

Shitara Y, Hirano M, Adachi Y, Itoh T, Sato H, and Sugiyama Y (2004) In vitro and in vivo correlation of the inhibitory effect of cyclosporin A on the transporter-mediated hepatic uptake of cerivastatin in rats. Drug Metab Dispos 32:1468-1475.

Tsujihata Y, Ito R, Suzuki M, Harada A, Negoro N, Yasuma T, Momose Y, and Takeuchi K (2011) TAK-875, an orally available $\mathrm{G}$ protein-coupled receptor $40 /$ free fatty acid receptor 1 agonist, enhances glucose-dependent insulin secretion and improves both postprandial and fasting hyperglycemia in type 2 diabetic rats. $J$ Pharmacol Exp Ther 339:228-237.

van Staden CJ, Morgan RE, Ramachandran B, Chen Y, Lee PH, and Hamadeh HK (2012) Membrane vesicle ABC transporter assays for drug safety assessment. Curr Protoc Toxicol 54: 23.5.1-23.5.24. Wilby AJ, Maeda K, Courtney PF, Debori Y, Webborn PJ, Kitamura Y, Kusuhara H, Riley RJ, and Sugiyama Y (2011) Hepatic uptake in the dog: comparison of uptake in hepatocytes and human embryonic kidney cells expressing dog organic anion-transporting polypeptide 1B4. Drug Metab Dispos 39:2361-2369.

Wolenski FS, Zhu AZX, Johnson M, Yu S, Moriya Y, Ebihara T, Csizmadia V, Grieves J, Paton M, Liao M, et al. (2017) Fasiglifam (TAK-875) alters bile acid homeostasis in rats and dogs: a potential cause of drug induced liver injury. Toxicol Sci 157:50-61.

Address correspondence to: Akifumi Kogame, 26-1, Muraoka-Higashi 2-Chome, Fujisawa, Kanagawa 251-0012, Japan. E-mail: akifumi.kogame@takeda.com 